$\overline{\text { Original }}$

\title{
Sterilization Efficiency of the Photocatalyst against Environmental Microorganisms in a Health Care Facility
}

\author{
HIDEHARU SHINTANI ${ }^{*}$, SINOBU KUROSU ${ }^{2}$, AKIKO MIKI ${ }^{2}$, \\ FUMIE HAYASHI ${ }^{3}$, AND SHIGEKAZU KATO ${ }^{4}$ \\ 'National Institute of Health Sciences, 1-18-1 Kamiyoga, Setagaya, Tokyo 158-8501, Japan, \\ ${ }^{2}$ Minofagen Pharmaceutical Co., Ltd., Zama Factory, 2-2-3, Komatsubara, Zama, \\ Kanagawa 228-0002, Japan, ${ }^{3}$ Namiki Clinic, 2-10-22 Uchidabashi Minami, \\ Nagoya 457-0862, Japan, ${ }^{4}$ Photo-Catalytic Materials Institute, 400 \\ Iwasaki, Komaki, Aichi 485-0011, Japan
}

Received 21 July 2005/Accepted 30 November 2005

The photocatalyst equipment consists of a titanium dioxide membrane and an ultraviolet lamp. The authors studied if the photocatalyst equipment is practically useful in sterilizing environmental microorganisms in the health care facility. The number of microorganisms was compared in the cases of no sterilization (control) and the photocatalyst sterilization. As a result, a statistical difference was observed between control and the photocatalyst sterilization against airborne microorganisms $(p<0.01)$, but not against surface microorganisms $(p>0.2)$. The photocatalyst uses an air sucking system, so it may be ineffective against microorganisms tightly attached to surfaces. However, the effectiveness of the photocatalyst to sterilize airborne microorganisms in the health care facility was successfully confirmed. Concerning the humidity effect on the photocatalyst sterilization, the authors compared the number of airborne microorganisms in cases of the control, UV alone and photocatalyst sterilization when humidity was changed. A statistical difference was observed between UV and the photocatalyst sterilization $(p<0.01$ ) when humidity was increased to $60-70 \%$, but not observed between UV and the photocatalyst sterilization ( $p>0.2$ ) when humidity was not controlled and was around $10-20 \%$. This indicates that maintaining high humidity levels will present satisfactory sterilization results due to a greater production of $\mathrm{OH}$ radicals. From data obtained, no effect of the adsorption on the $\mathrm{TiO}_{2}$ membrane could be observed.

Key words : Photocatalyst/Nosocomial infection/Titanium dioxide/Environmental microorganisms/ Humidity

\section{INTRODUCTION}

Nosocomial infections are recently a great problem in the health care facilities (Sahini-Kardasi, 1996; Barbeau et al., 1998; Weist et al., 2002; Harthug and Lingaas, 2002; Vincent, J, 2002; Miki et al., 2003a,b; Sakurai et al., 2003; Shintani et al., 2004). Nosocomial infections have been reported to be mostly due to cross-transmission via hands from

${ }^{*}$ Corresponding author. Tel: $+81-3-3700-9268$, Fax : $+81-$ 3-3707-6950. health care employees to immunodeficient patients (Sahini-Kardasi, 1996; Weist et al., 2002), but this has not been completely defined yet. It is confirmed that washing hands frequently is important ways to prevent nosocomial infections in the health care facility. In addition it is indispensable to sterilize airborne and surface microorganisms to diminish nosocomial infections. These airborne and surface microorganisms mostly come from humans (Barbeau et al., 1998; Miki et al., 2003a,b). The representative microorganisms related to nosocomial infections have been presented by Barbeau et al. (1998).

According to reports, several sorts of microor- 
ganisms and viruses in plates were exposed to the photocatalyst in a model experiment and satisfactory sterilization results were obtained (Ireland et al., 1993; Maness et al., 1999; Huang et al., 2000; De Heredia et al., 2001; Wolfrum et al., 2002). However, the sterilization efficiency of the photocatalyst in the practical use with regard to environmental microorganisms in places such as in the health care facility the validation study of the photocatalyst sterilization has not yet been conducted. It is keenly required to keep the environment of the health care facility clean to prevent nosocomial infections. Prior to maintaining cleanliness within the health care facility with the use of the photocatalyst, it is necessary to confirm whether the photocatalyst equipment may be practically useful for sterilizing the environmental microorganisms in the health care facility.

In addition the effect of humidity on the photocatalyst sterilization has not been reported so far. Humidity is thought to play an important role in the photocatalyst sterilization with regards to $\mathrm{OH}$ radical production.

The photocatalyst equipment in general consists of an ultraviolet (UV) lamp and an anatase type titanium dioxide $\left(\mathrm{TiO}_{2}\right)$ membrane (Miki et al., 2003b). With $\mathrm{UV}$ irradiation onto the $\mathrm{TiO}_{2}$ membrane, $\mathrm{OH}$ radicals are produced (Shibata et al., 1998; Kashige et al., 2001). The produced $\mathrm{OH}$ radical is of major importance in this sterilization system. The $O$ radical or $\mathrm{OOH}$ radial is also produced from the irradiated $\mathrm{TiO}_{2}$ membrane, but the sterilization efficiency of the $\mathrm{OH}$ radical is superior to that of $\mathrm{O}$ radical or $\mathrm{OOH}$ radical. The role of the $\mathrm{OH}$ radical in sterilization is the proton $\left(\mathrm{H}^{+}\right)$removal from the microorganisms, which causes the oxidation of the microorganisms and results in successful sterilization. The $\mathrm{OH}$ radical itself changes to $\mathrm{H}_{2} \mathrm{O}$ by the reaction of $\mathrm{OH}^{*}+\mathrm{H}$ (Shibata et al., 1998; Kashige et al., 2001).

When the air of the environment contaminated with microorganisms is sucked into the photocatalyst equipment, the microorganisms within the photocatalyst will be sterilized with the combined effect of UV irradiation and $\mathrm{OH}$ radical exposure produced from the irradiated $\mathrm{TiO}_{2}$. The major UV sterilization mechanism was known as the thymine dimer, thymine-cytosine dimer or cytosine-cytosine dimer formation, which depends on wavelength (Setlow, 1966; Setlow and Carrier, 1966; Yasui and McCready, 1998). However, UV sterilization efficiency with regard to fungi was not satisfactory. Thus it is speculated that when $\mathrm{UV}$ and $\mathrm{OH}$ radical treatment are combined, the synergistic effect can produce more successful sterilization results with regard to bacteria and fungi. To confirm this, the authors compared the number of the airborne and surface microorganisms receiving no sterilization (control) and photocatalyst sterilization treatment. The results were analyzed using several statistical procedures.

It has not yet additionally been confirmed how photocatalyst function in sterilization may change when the humidity around the photocatalyst equipment changes. However, it was speculated that it would be important to maintain relatively high level of humidity to attain satisfactory and reproducible sterilization results with the photocatalyst equipment. That is because the $\mathrm{OH}$ radical is from water (humidity), so it can be speculated that the photocatalyst efficiency might increase when humidity increases. To confirm this, the author studied the sterilization efficiency of the photocatalyst against airborne microorganisms with different levels of humidity around the photocatalyst equipment. These results are reported herein.

\section{MATERIALS AND METHOD}

The experiment was conducted three times on three different days during winter when air is dry (humidity was around $10-20 \%$ or less). Five places in the dialysis room were sampled for the airborne and the surface microorganisms for each experiment (Figure 1). Two photocatalyst equipments were set beside opposite walls (Figure 1). The patients in the dialysis room were all the same during these experiments. The number of patients was six. The health care facility at which the experiment was conducted was the dialysis room of Namiki Clinic in Nagoya, Japan (Shintani et al., 2004).

The experiment was conducted without sterilization as a control and with sterilization with the photocatalyst equipment to compare the photo-

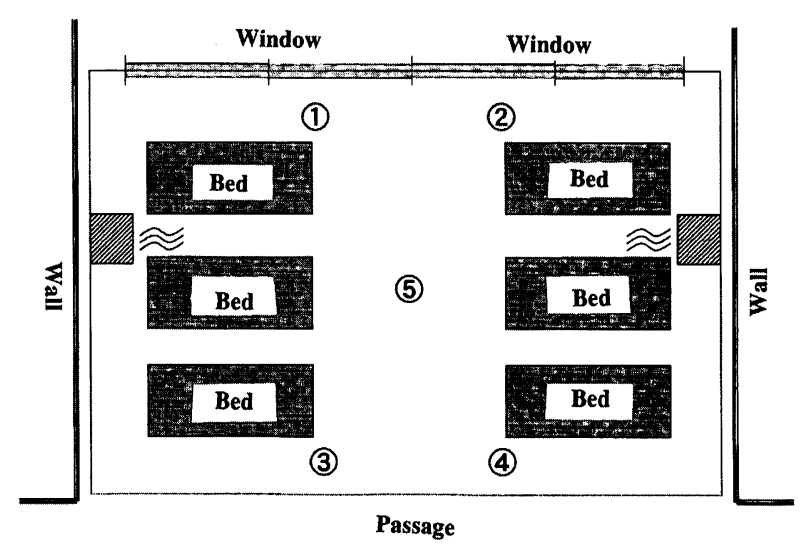

FIG. 1. Sampling position for airborne and surface microorganisms in the dialysis room. 
catalyst efficiency in terms of the resulting CFU (colony forming unit) of airborne and surface microorganisms. For the humidity confirmation, experiments used three sorts of treatment conditions: control (no sterilization), UV alone and photocatalyst sterilization (UV $+\mathrm{TiO}_{2}$ membrane exposed to UV). These experiments were repeated three times on different days $(n=3)$.

The photocatalyst equipment used consists of the UV $\left(253.7 \mathrm{~nm}\right.$ ) lamp and the anatase type $\mathrm{TiO}_{2}$ membrane filter. The specific photocatalyst equipment used in this experiment was Plesant ${ }^{R}$ from the PhotoCatalytic Materials Institute in Nagoya, Japan. The description of Plesant ${ }^{R}$ is presented in Table 1. The photocatalyst equipment was operated for whole days during the experiment.

The airborne and surface microorganisms were determined and identified. These microorganisms were bacteria including MRSA, vegetative cells and spore formers, fungi, mold and yeast.

The biochemical identification of the airborne and the surface microorganisms was conducted with both $\mathrm{API}^{\mathrm{R}}$ (Biologue Co., Ltd., Lion, France) and BBL Crystal $^{R}$ (Becton Dickinson Co., Ltd, Loveton Circle, Md) (Miki et al., 2003a,b; Shintani et al., 2004). When both results indicated the same microorganism, the result was considered valid, and otherwise defined to be invalid.

The number of colonies in the culture media were counted and expressed as CFU.

The airborne microorganisms were determined using the air sampler of SAS Super $100 \mathrm{CR}^{R}$ from PBI Co. Ltd (Milano, Italy) (Shintani et al., 2004). The function of SAS Super $100 \mathrm{CR}^{\mathrm{R}}$ has been described in the paper by Shintani et al. (2004). The bacterial and fungal microorganisms were trapped into $90 \mathrm{~mm}$ plates containing two sorts of culture media, individually, as described below.

The air sampler was used at $100 \mathrm{~L} / \mathrm{min}$ for three minutes at five different points in the dialysis room on different five days (Figure 1). It means, as a whole, $4.5 \mathrm{~m}^{3}$ air was sucked, so that the colony number unit was CFU/4.5 $\mathrm{m}^{3}$.

The trapping and culture medium used for the de-

TABLE 1. The description of Plesant ${ }^{R}$

\begin{tabular}{ll}
\hline Type number & PL2003 \\
Battery source & AC100V \\
The volume of mind & maximum $3.5 \mathrm{~m}^{3} / \mathrm{min}$ \\
Noise & maximum $49 \mathrm{~dB}$ \\
Efficient surface area & maximum $25 \mathrm{~m}^{2}$ \\
Light source & UV lamp $6 \mathrm{~W} \times 4$ \\
Power used & maximum $55 \mathrm{~W}$ \\
Size $(\mathrm{mm})$ & $180 \times 430 \times 580$ \\
\hline
\end{tabular}

termination and the identification of the airborne bacteria was SCDALP (soybean casein digest agar lecithin polysorbate) from Difco Co., Ltd. (Becton Dickinson Co., Ltd.). The culture medium used for the airborne fungi was SDA (Sabouraud dextrose agar) from Difco Ltd. The cultivation of the airborne bacteria and fungi was conducted for 5 consecutive days at $30^{\circ} \mathrm{C}$ and 7 days at $25^{\circ} \mathrm{C}$, respectively (Miki et al., 2003a,b; Shintani et al., 2004). As SCDALP was confirmed to be superior to SCDA (soybean casein digest agar) in terms of the CFU of the growing microorganisms, SCDALP was selected (Shintani et al., 2004).

The sampling of the surface microorganisms was conducted with the stamping procedure using RODAC $^{R}$ plates from Becton Dickinson Co., Ltd. The surface bacteria was sampled by $R O D A C^{R}$ plate SCDALP and incubated for 5 consecutive days at 30 ${ }^{\circ} \mathrm{C}$ (Miki et al., 2003). The determination of the surface fungi was conducted using RODAC ${ }^{R}$ plate CPSDA (Chloramphenicol added Sabouraud dextrose agar) and incubated for 7 consecutive days at $25^{\circ} \mathrm{C}$ (Miki et al., 2003a,b). The cultivation efficiency between SDA and CPSDA for fungi growth indicated no significant difference. The number of plates used in this experiment was 15 plates, so the colony number unit was CFU/15plates.

The surface MRSA (Methicillin-resistant Staphylococcus aureus) was sampled using Petancheck ${ }^{R} 25$ MRSA from Eiken Co., Ltd. (Tokyo, Japan) and incubated for 5 consecutive days at $30^{\circ} \mathrm{C}$ (Miki et al., 2003a). MRSA was additionally identified using Denka Seiken MRSA kit (Tokyo, Japan), in the case when bacterial growth was observed in the selective MRSA culture medium from Eiken Co. Ltd. When both results were positive, the bacterium was defined as MRSA, and otherwise defined as negative (Miki et al., 2003a).

The humidifier (Matsushita Co. Ltd., FE-14KFW, Osaka, Japan) was set in front of sucking port of the photocatalyst equipment and the humidity at exhaust port of the photocatalyst equipment was determined. The effect of humidity on the photocatalyst in the sterilization of airborne microorganisms was studied.

The $D$ (decimal reduction) value of several sorts of microorganisms exposed to UV (253.7 nm) lamp was calculated with the fraction negative method (Stumb-Murphy-Cochran, Shintani et al., 1995b).

Radicals produced after UV exposure were determined by using ESR (electron spin resonance) of JES-FE1XG (JEOL of Nihon denshi Co., Ltd., Tokyo, Japan). 


\section{RESULTS}

\section{Airborne vs. surface microorganisms}

The numbers of the airborne microorganisms and the surface microorganisms under conditions of no sterilization and the photocatalyst sterilization are presented in Tables 2 and 3, respectively. The representative identified airborne and surface microorganisms in the dialysis room of the Namiki Clinic are presented in Tables 4 and 5, respectively. These results resembled those reported previously (Shintani et al., 2004). This means identical microorganisms almost always exist in the same place due to the presence of almost identical employees. Airborne and surface microorganisms are mostly Staphylococcus spp., Micrococcus spp., Bacillus spp., and Corynebacterium spp. and their origins are mostly from humans (Barbeau et al., 1998; Miki et al., 2003a,b; Shintani et al., 2004). The ratios of identified airborne and surface microorganisms are presented in Figures 2 and 3 , respectively. The total CFU of the airborne microorganisms and that of surface microorganisms were around 300 and 40 (Tables 2, 3 ), respectively. The number of microorganisms to be successfully identified was around $60 \%$ of the total microorganisms sampled. The rest of $40 \%$ showed mismatches between $\mathrm{API}$ and $\mathrm{BBL}$ Crystal ${ }^{\mathrm{R}}$ results or

TABLE 2. Number of colonies in the case of no sterilization.

\begin{tabular}{|c|c|c|c|c|c|c|c|}
\hline \multicolumn{8}{|c|}{ Airborne microorganisms } \\
\hline \multirow{2}{*}{$\begin{array}{l}\text { Culture } \\
\text { medium }\end{array}$} & \multirow{2}{*}{$\begin{array}{l}\text { Determination } \\
\text { dala }\end{array}$} & \multicolumn{6}{|c|}{ Determination cycle (CFU) } \\
\hline & & No.1 & No.2 & No.3 & No.4 & No.5 & Subtotal \\
\hline \multirow[t]{4}{*}{ SDA $25^{\circ} \mathrm{C}$} & $1^{\text {st }}$ & 2 & 10 & 10 & 10 & 15 & 47 \\
\hline & $2^{\text {nd }}$ & 3 & 1 & 4 & 3 & 5 & 15 \\
\hline & $3^{\text {rd }}$ & 13 & 8 & 8 & 5 & 11 & 45 \\
\hline & & & & & Total & & 107 \\
\hline \multirow[t]{4}{*}{$\operatorname{SCDALP} 30^{\circ} \mathrm{C}$} & $1^{\text {st }}$ & 3 & 5 & 12 & 5 & 4 & 29 \\
\hline & $2^{\text {nd }}$ & 14 & 19 & 13 & 9 & 7 & 62 \\
\hline & $3^{\text {rd }}$ & 14 & 15 & 10 & 16 & 15 & 70 \\
\hline & & & & & Total & & 161 \\
\hline \multicolumn{8}{|c|}{ Surface microorganisms } \\
\hline \multirow{2}{*}{$\begin{array}{l}\text { Culture } \\
\text { medium Temperatur }\end{array}$} & Determination & & etermi & ination & n cycle & (CFL & \\
\hline & & No.1 & No.2 & No.3 & No.4 & No.5 & Subtotal \\
\hline \multirow[t]{4}{*}{$\overline{\mathrm{CPSDA}} 25^{\circ} \mathrm{C}$} & $1^{\text {st }}$ & 1 & 0 & 0 & 0 & 0 & 1 \\
\hline & $2^{\text {nd }}$ & 0 & 1 & 3 & 1 & 0 & \\
\hline & $3^{\text {rd }}$ & 0 & 0 & 2 & 0 & 0 & 2 \\
\hline & & & & & Total & & 8 \\
\hline \multirow[t]{4}{*}{$\overline{S C D A L P} 30^{\circ} \mathrm{C}$} & $1^{\text {st }}$ & 1 & 0 & 1 & 3 & 1 & \\
\hline & $2^{\text {nd }}$ & 0 & 0 & 4 & 2 & 0 & 6 \\
\hline & $3^{\text {rd }}$ & 2 & 1 & 2 & 1 & 0 & 6 \\
\hline & & & & & Total & & 18 \\
\hline
\end{tabular}

were unidentified microorganisms. Figures 2 and 3 present almost identical ratios results. This indicates that the origin of surface microorganisms is airborne microorganisms and airborne microorganisms are from humans.

The numbers of the airborne and surface microorganisms found under no sterilization (control) and the photocatalyst sterilization conditions are presented in Figures 4 and 5, respectively.

Photocatalyst sterilization was effective in diminishing the airborne microorganisms of bacteria and fungi $(p<0.01$, Figure 4$)$. On the contrary, it was ineffective against surface microorganisms ( $p>0.2$, Figure 5 ). This was due to the mechanism of photocatalyst equipment, Plesant ${ }^{R}$. The air sucking system in the photocatalyst equipment, Plesant ${ }^{R}$, may not work effectively against microorganisms tightly attached to surfaces and may only be effective against the air-

TABLE 3. Number of colonies in the case of photocatalyst sterilization.

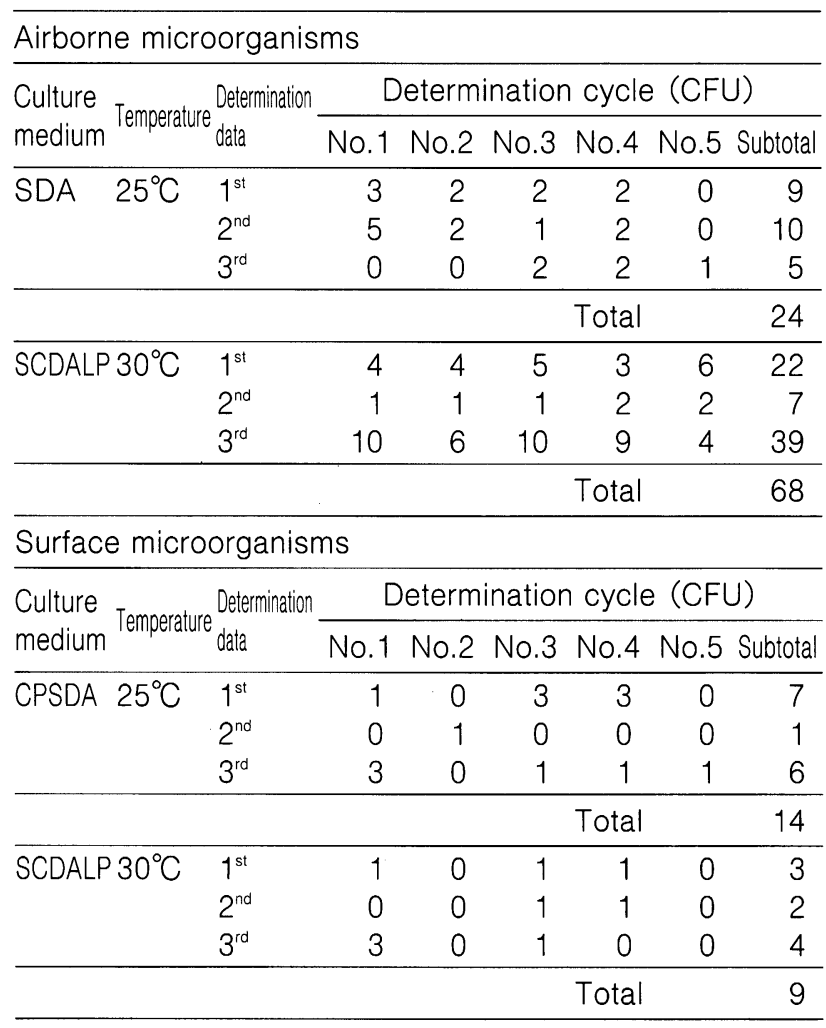

TABLE 4. Representative airborne microorganisms found in the dialysis room.

Micrococcus luteus, M. sedentarius, M. Iylae

Bacillus cereus, B. brevis, B. megaterium

Sphingomonas paucimobilis

Staphylococcus hominis, S. schliferi, S. kloosil, S. aureus, S. haemolytios, S. saprophytious

Corynebacterium aquaticum 
TABLE 5. Representative surface microorganisms found in the dialysis room

Staphylococcus haemolytious, S. chnii, S. hominis, S. intermedius, S. epidermidis, S. xylosus, S. lentus, $S$. lugdunensi, S. capitis, S. saprophytious, S. schliferi, S. cohnii

Micrococcus kristinae, M. luteus, M. sedentarius, M. Iylae, M. roseus

Bacillus cereus, B. subtilis, B. megaterium, B. licheniformis Sphingomonas paucimobilis

Acinetobacyer Iwoffi

Streptpcoccus sangunis, S. vestibularis, S. schleiferi

Bergeyella zoohelcum

Aerococcus urinae

Chrysobacterium indologens

Brevandimonas vesicularis

Corynebacterium aquaticum

Burkholderia cepacia
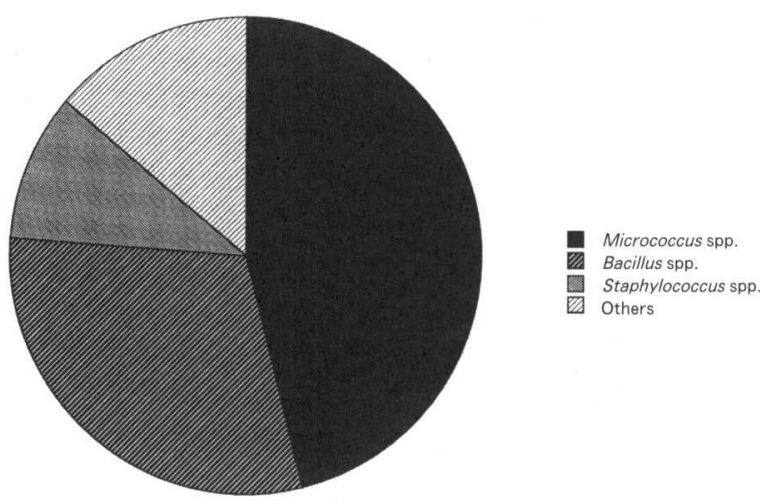

FIG. 2. The ratios of bacteria, fungi and yeast and so on for airborne microorganisms detected in the dialysis room

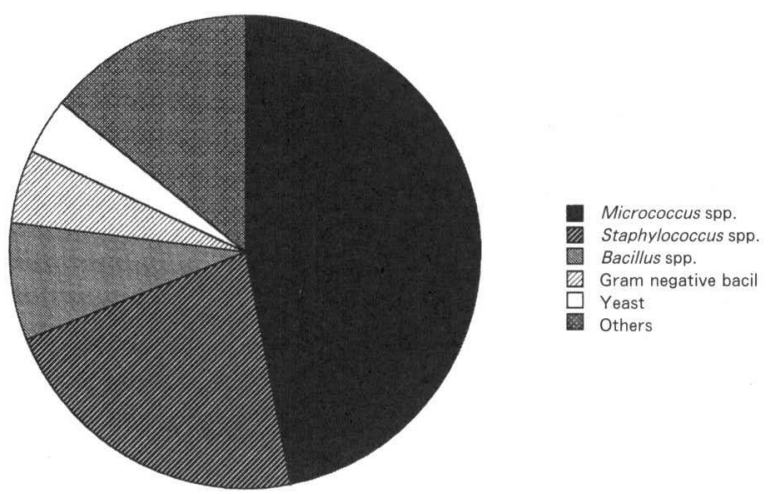

FIG. 3. The ratios of bacteria, fungi and yeast and so on for surface microorganisms detected in the dialysis room

borne microorganisms. The stay within the photocatalyst chamber was only a few seconds and this period was too short to attain a satisfactory sterilization effect.

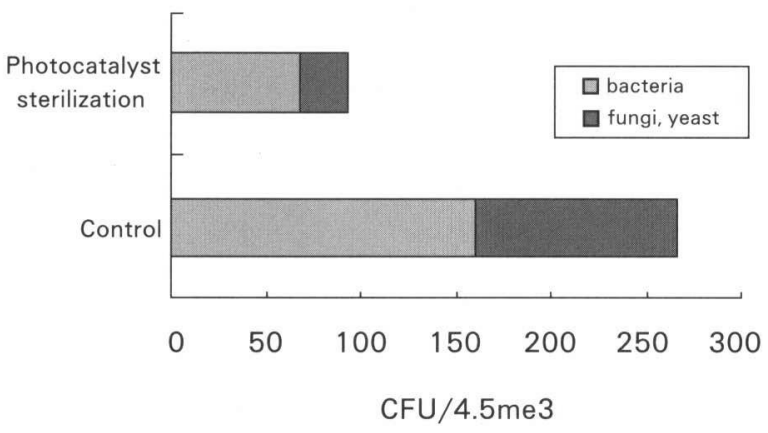

FIG. 4. The comparison of airborne bacteria and fungi in the case of no sterilization and photocatalyst sterilization for bacteria and fungi.

Unit: CFU/ $4.5 \mathrm{~m}^{3}$

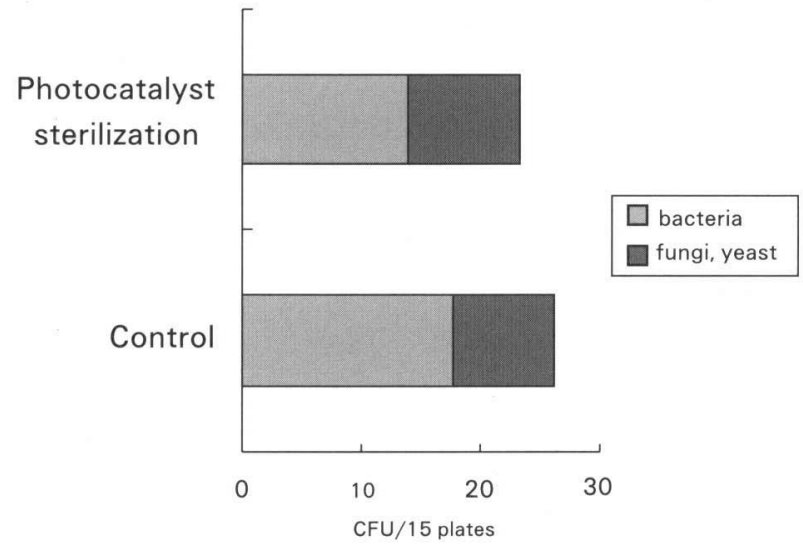

FIG. 5. The comparison of surface bacteria and fungi in the case of no sterilization and photocatalyst sterilization for bacteria and fungi.

Unit: CFU/15 plates

\section{Humidity effect}

The number of the airborne microorganisms with the control, UV and of the photocatalyst sterilization without humidity control is presented in Figure 6 (humidity was around 10-20\%). Figure 7 presents the sterilization effect when humidity was maintained at $60-70 \%$ around the photocatalyst equipment.

Figures 6 and 7 indicate that microorganisms were effectively sterilized by the photocatalyst or UV exposure treatment when compared with the control. This result was the same with or without humidity control.

According to the statistical analysis, photocatalyst sterilization was more effective in sterilizing the airborne bacteria and fungi $(p<0.01$, Figure 7$)$ compared with UV irradiation alone when the humidity was maintained at around 60 to $70 \%$. On the contrary, photocatalyst sterilization was not so much more effective in sterilizing the airborne bacteria and 


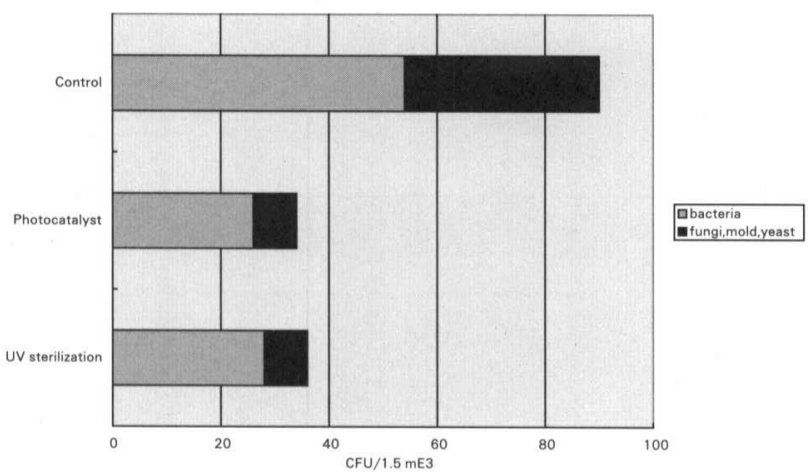

FIG. 6. Results from the control, photocatalyst and UV sterilization of airborne bacteria and fungi when humidity was not controlled.

Humidity was around $10-20 \%$

Unit: CFU/1.5m ${ }^{3}$

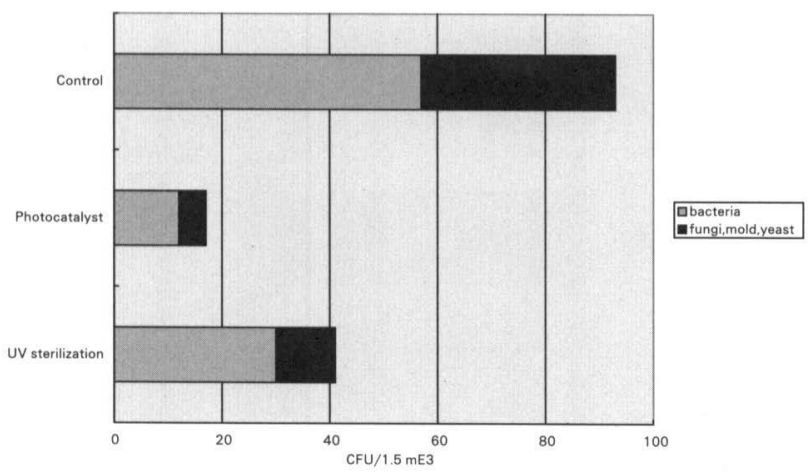

FIG. 7. Results from the control, photocatalyst and UV sterilization of airborne bacteria and fungi when humidity was controlled.

Humidity was around $60-70 \%$

Unit: CFU/1.5m

fungi compared with UV irradiation alone when humidity was not controlled (humidity was 10-20\%) ( $p>0.2$, Figure 6).

\section{DISCUSSION}

\section{Airborne vs. surface microorganisms}

UV irradiation sterilization was mainly surface sterilization and not always sufficiently effective against fungi, but effective against bacteria including spore formers, i.e. Bacillus spp. and Clostridium spp. The D values of several microorganisms with regard to UV irradiation at $253.7 \mathrm{~nm}$ are presented in Table 6 .

Most bacteria were sterilized by UV irradiation because they were sensitive to UV irradiation (Table 6). The fungi most tolerant to UV irradiation sterilization was Aspergillus niger (Table 6). This was due to the black color of the appearance, thus it is considered as a biological indicator of UV sterilization and pulsed light sterilization.

The mechanism of UV sterilization is the dimer formation of DNA or RNA base, i.e. thymine-thymine dimer, thymine-cytosine dimer or cytosine-cytosine dimer formation, which results in the disorder of DNA message to fabricate proteins though messenger RNA (Setlow, 1966; Setlow and Carrier, 1966; Yasui et al., 1998).

Our speculation is that fungi tolerant to UV sterilization will be better controlled when $\mathrm{OH}$ radicals from irradiated $\mathrm{TiO}_{2}$ membrane are combined with UV irradiation. The combined effect of $\mathrm{UV}$ and $\mathrm{OH}$ radical irradiation exposure was speculated to successfully sterilize both bacteria and fungi simultaneously. This speculation seemed correct for airborne microorganisms (Figure 4, $p<0.01$ ), but was not always correct for surface microorganisms (Figure $5, p>0.2$ ). The inferior effect on the latter was perhaps due to the photocatalyst equipment function itself. This equipment was not efficient against microorganisms tightly attached to surfaces and as a result they were not adequately sucked into the photocatalyst equipment of Plesant ${ }^{\mathrm{R}}$. However, this system is favorably safe for the prevention of UV and $\mathrm{OH}$ radical exposure to hu-

TABLE 6. Decimal reduction value (D) of bacteria, fungi and yeast by $U V$ radiation at $253.7 \mathrm{~nm}$.

\begin{tabular}{lc}
\hline Microorganisms & D value $\left(\mathrm{mW} \cdot \mathrm{s} / \mathrm{cm}^{2}\right)$ \\
\hline Aspergillus niger & 87.3 \\
A. flavus & 39.7 \\
A. glaucas & 29.3 \\
Rhizopus spp & 74.7 \\
Penicilium digitatum & 30.1 \\
P. expansum & 7.2 \\
P. roquefori & 9.6 \\
Mucor spp & 10.3 \\
Bacillus subtilis (spore) & 11.1 \\
B. mesentericus (spore) & 9.6 \\
Streptococcus pyrogenes & 3.2 \\
Staphylococcus albus & 3.2 \\
Staphylococcus aureus & 3.2 \\
Enterococci & 4.8 \\
Escherichia coli & 2.4 \\
Vibrio cholerae & 2.4 \\
Salmonella enterica & 4.8 \\
Pseudomonas aeruginosa & 3.2 \\
Shigella dysenteriae & 1.6 \\
Salmonella typhimurium & 1.6 \\
Saccaromyces cerevieciae & 4.0 \\
Saccaromyces bayanus & 4.8 \\
Saccaromyces sake & 11.3
\end{tabular}

$\mathrm{mW} \cdot \mathrm{s} / \mathrm{cm}^{2}$ is a unit of the incident energy. 
mans, or it would otherwise be dangerous to the humans around the photocatalyst equipment. Within the photocatalyst equipment, the UV lamp is placed in the center and it is surrounded with $\mathrm{TiO}_{2}$ membranes. The air sucked inside is passed through the area between UV lamp and $\mathrm{TiO}_{2}$ membrane for a few seconds. During this time, microorganisms are sterilized by the combined effect of $\mathrm{OH}$ radicals and UV irradiation exposure. The leaking out of the UV light and $\mathrm{OH}$ radicals produced prevented in the photocatalyst equipment of Plesant ${ }^{R}$. This indicates that it is totally safe for humans around the equipment.

The sterilization efficiency of the anatase type $\mathrm{TiO}_{2}$ membrane of photocatalyst equipment was significant in practical use as well as in model experiment (Ireland et al., 1993; Shibata et al., 1998; Yasui et al., 1998; Maness et al., 1999; Huang et al., 2000; De Heredia et al., 2001; Kashige et al., 2001; Wolfrum et al., 2002). However, as shown in Figures 4 and 5, some bacteria and fungi were found after photocatalyst sterilization. However, there was no way to distinguish whether these microorganisms were survivors of the photocatalyst sterilization or new comer microorganisms entering the dialysis room together with humans. This was because the dialysis room was not closed during the experiment, but open to the entry of many peoples who are the origin of the environmental microorganisms in the dialysis room. Our experiment was done in the open room as usual.

When the data for airborne microorganisms in Tables 2 and 3 were examined, it was found that the vertical sum of each determination cycle was almost identical, which mean the determination variation within the cycle run was not significant even if the experiment was conducted in an open room. As the data of surface microorganisms was not quantitative, it could not be clearly evaluated in comparison with that of airborne microorganisms, but almost the identical phenomenon could be found as for the airborne microorganisms.

If the photocatalyst sterilization is insufficient, the diseases associated with fungi must be seriously discussed at health care facilities because most of patients show an insufficient or lacking immune function. One disease associated with fungi is the Aspergillus syndrome (Hodgson and Flannigan, 2001). It mainly occurs in the lung of immunodeficient patients. Another example is the deterioration of food with Aspergillus flavus or A. parasiticus. These fungi produce aflatoxin, which causes cancer when patients and healthy people eat the foods spoiled with these fungi. Therefore, these fungi must be completely disinfected in the health care facility for the benefit of patients as well as health care employees.

The distribution of the airborne microorganisms was not a normal distribution, but the Poisson distribution (Miki et al., 2003b; Shintani et al., 2004). In order to approximate the Poisson distribution to the normal distribution, the raw data of the number of microorganisms (Tables 2 and 3 ) were transformed to the logarithm values or the square root values. These were conducted the statistical analysis of the Student $t$ test and/or ANOVA (analysis of variation). When the data was transformed to the logarithm values, zero data was out of value. In order to avoid data that was out of value, the square root transformation was mostly adapted to conduct Student $t$ test.

The $p$ values obtained by the paired Student $t$ test for the airborne microorganisms and the surface microorganisms are presented in the text. The Student $t$ test results from the data transformed to the logarithm values were almost identical to those from the square root transformed data. In addition, the ANOVA results were also identical to Student $t$ test results with only one exception, indicating that both phenomena are identical. A statistically significant difference can be observed for airborne microorganisms subjected to no sterilization (control) and photocatalyst sterilization treatments $(p<0.01$, Figure 4$)$, but was not observed for the surface microorganisms ( $p>0.2$, Figure 5). This was the same as in the case of the surface MRSA ( $p>0.2$, data not shown).

The single exposure period within the photocatalyst equipment was for a few seconds. UV irradiation exposure even for a few seconds is effective against bacteria and yeast, but not fungi (Takeshita et al., 2003). According to model experiments using the direct exposure with photocatalyst for 6 hours to bacteria or virus in the plates (Ireland et al., 1993; Maness et al., 1999; Huang et al., 2000; De Heredia et al., 2001; Wolfrum et al., 2002), total death results were obtained. According to our result using the Plesant ${ }^{R}$, the sterilization effect on the surface microorganisms (Figure 5) significantly differed from that on the airborne microorganisms (Figure 4). In model experiment, fungi were not tested and exposure period in our experiment and that in model experiment were significantly different (a few seconds vs. 6 hours). According to the current results obtained so far, the photocatalyst equipment of Plesant ${ }^{R}$ cannot be recommend for use for the purpose of sterilizing surface microorganisms.

Prior to the experiment, the authors speculated that the number of fungi with no sterilization (control) would be less than that with the photocatalyst sterilization. The speculation is based on the combined effect of $\mathrm{OH}$ radicals and UV irradiation. This proved to 
be correct for airborne microorganisms (Figure 4), but not always correct for surface microorganisms (Figure 5). Therefore, as an alternative way to sterilize the surface microorganisms, it may be necessary to consider what was done in model experiment with the direct exposure to the photocatalysts (Ireland et al., 1993; Maness et al, 1999; Huang et al., 2000; Wolfrum et al., 2002). Or direct exposure with sunlight to the wall or floor surface immobilized $\mathrm{TiO}_{2}$ to sterilize surface microorganisms. However, model procedure and direct exposure to surface immobilized $\mathrm{TiO}_{2}$ have a possibility to be exposed to $\mathrm{OH}$ radicals directly. Therefore they are risky and such direct exposure is not totally recommended.

To improve the sterilization efficiency of the photocatalyst equipment used in the experiment, there may exist several things to consider for innovation. As the origin of $\mathrm{OH}$ radical is water (humidity), the sufficient supply of water to the $\mathrm{TiO}_{2}$ membrane is indispensable to improve the sterilization results of the photocatalyst. The experiment was conducted in winter, the dry season in Japan, and in addition the air conditioner was used in the dialysis room without any use of a humidifier. During the winter season, the humidity in the health care facility is around 10 to $20 \%$ or less. The insufficient water content may result in the insufficient function of the $\mathrm{TiO}_{2}$ membrane to produce the sufficient quantity of $\mathrm{OH}$ radicals for attaining satisfactory photocatalyst sterilization results. If the humidity level differs, the results may also differ. In that sense, it would be desirable to equip a humidity controller in the photocatalyst equipment to attain a successful and reproducible sterilization result, otherwise any seasonable variation of the photocatalyst sterilization may be observed in addition to the unsatisfactory sterilization result.

Based on the above speculations, an experiment to confirm the effect of humidity on sterilization efficiency of the photocatalyst was conducted as follows.

\section{Humidity effect}

The UV irradiation sterilization was mainly surface sterilization and not always sufficiently effective against fungi (Table 6), but effective against bacteria including spore formers, i.e. Bacillus spp. and Clostridium spp. Fungi is most tolerant to UV sterilization than bacteria including spore formers (Table 6). Fungi tolerate UV irradiation sterilization compared with bacteria including spore formers. The fungus most tolerant to UV irradiation sterilization was Aspergillus niger. The next fungus most tolerant to UV was Rhizopus spp (Table 6).

UV or pulsed light irradiation alone is insufficient to sterilize fungi (Dunn et al., 1997; Feldman, 1997; Fine, and Gervais, 2004). In that sense, the combined effect of $\mathrm{OH}$ radical and UV exposure was necessary, which is the same as reported for the combined effect of UV irradiation and $\mathrm{H}_{2} \mathrm{O}_{2}$ gas exposure (Imlay and Linn, 1988). The speculation regarding the $\mathrm{OH}$ radical contribution in the photocatalyst sterilization was correct. However, this speculation was correct only when there was sufficient humidity. When humidity was insufficient, the $\mathrm{OH}$ radical effect was unsatisfactory for the photocatalyst sterilization. This was due to the insufficient production of $\mathrm{OH}$ radicals from water. This was statistically confirmed from the comparison of UV vs. photocatalyst sterilization when humidity was sufficient (Figure 7,60-70\%) and insufficient (Figure 6, 10-20\%). Maintaining the humidity at a higher degree is an essential task to attain significant and reproducible photocatalyst efficiency to avoid any seasonable variation in performance.

From the comparison between Figures 6 and 7, sufficient humidity was indispensable to attain satisfactory sterilization effect against bacteria and fungi. UV sterilization efficiency was reported to decrease when the humidity is high (Ko et al., 2000), but the increase in $\mathrm{OH}$ radicals by increasing humidity could overcome the reported diminishment of UV irradiation efficiency and a desirable sterilization result was obtained (Figure 7). This indicates that the interference of humidity in UV sterilization was not significant contrary to the results reported by Ko et al (2000).

To conduct the statistical analysis of data in Figures 6 and 7, data were transformed to the square root value to perform the Student $t$ test, ANOVA (analysis of variation) and/or $\chi^{2}$ test because the distribution of the environmental microorganisms is the Poisson distribution. The $p$ value determined by the paired Student $t$ test for the airborne microorganisms using the square root value was conducted and presented in the text. The Student $t$ test result from the data transformed to the square root values and that of ANOVA result was identical. The result of $\chi^{2}$ test was somewhat different from that of Student $t$ test and ANOVA, however a tendency is identical. A statistically significant difference could be observed between the airborne microorganisms subject to the control and photocatalyst treatments $(p<0.01$, Figures 6 and 7 ). On the contrary, no statistical difference $(p>0.2)$ could be observed between photocatalyst and UV sterilization in Figure 6 for both bacteria and fungi when the humidity was not controlled. However, a statistical difference $(p<0.01)$ in bacteria and fungi could be observed between those receiving photocatalyst and UV exposure in Figure 7 when humidity was maintained at $60-70 \%$. The 
photocatalyst sterilization performed well even though the UV function is thought to be somewhat decreased at high humidity as reported by Ko et al (2000). However, it is speculated that humidity at more than $70 \%$ would show inferior results to those obtained at $60-70 \%$ due to insufficiency of UV function as reported by Ko et al (2000).

In addition, no significant difference in the CFU from air samples could be observed between the control and the photocatalyst equipment turned off UV irradiation. (data not shown). This indicates the adsorption phenomena of environmental microorganisms onto the $\mathrm{TiO}_{2}$ membrane with ion exchange, ion bonding, hydrogen bonding or Van der Walls bonding is not significantly observed. This can also be explained from the comparison between photocatalyst and UV irradiation in Figure 6 compared with that in Figure 7. The reason for the decrease in the microbiological number by photocatalyst treatment in Figure 7 is not due to increasing adsorption, but increasing $\mathrm{OH}$ radicals by increasing humidity, which results in satisfactory sterilization. In addition, if adsorption phenomenon was significant, it could not be explained no significant difference between UV alone (no membrane) and photocatalyst $\left(\mathrm{TiO}_{2}\right.$ membrane exists) in Figure 6.

Even though $\mathrm{OH}$ radicals cannot be directly determined with ESR or chemiluminescence (Shintani, 1995) as $\mathrm{OH}$ radicals exist in the interior of the photocatalyst equipment, we can speculate that the $\mathrm{OH}$ radicals will increase within the photocatalyst with increasing humidity. This is because we determined stable radicals after photocatalyst sterilization and obtained the result that the stable radicals increased at higher humidity by ESR compared with fewer stable radicals at lower humidity levels. It is necessary to note that the life period of active radicals is quite short and the produced amount of the active $\mathrm{OH}$ radicals is not abundant. In addition, the production of stable radicals by increasing the humidity may not always parallel the produced amount of active $\mathrm{OH}$ radicals. It may be sure that active $\mathrm{OH}$ radicals will increase with increasing humidity up to 60 to $70 \%$. In addition, in every experiment conducted herein, there was no data supporting the adsorption phenomenon of environmental microorganisms on any membranes used in the photocatalyst sterilization, thus the adsorption phenomenon onto membrane can be denied in the photocatalyst function.

From the result described so far, it was clarified that sufficient humidity for the photocatalyst equipment improves the sterilization efficiency. Humidity is an indispensable factor to increase the efficiency of the photocatalyst function; which is the same as in the cases of peracetic acid gas (Shintani, 2002; Shintani, 2003; Omori et al., 2005), ozone gas sterilization (Sakurai et al., 2003) and ethylene oxide sterilization (Shintani, et al., 2005).

The single exposure period of airborne microorganisms to $\mathrm{OH}$ radicals within the photocatalyst is around a few seconds. It must be lengthened to attain greater sterilization efficiency. In addition, $\mathrm{OH}$ radicals from the irradiated $\mathrm{TiO}_{2}$ must be increased to improve the sterilization effect of the photocatalyst on airborne microorganisms.

By lengthening the exposure period within the chamber and increasing the $\mathrm{OH}$ radical formation, photocatalyst sterilization efficiency may be much improved and as a result nosocomial infectious disease in the health care facility may be significantly decreased in future when the improved photocatalyst equipment becomes available.

\section{REFERENCES}

Barbeau, J., Gauthier, C., and Payment, P. (1998) Biofilms, infectious agents, and dental unit waterlines. Can. J. Microbiol., 44, 1019-1028.

De Heredia, J. B., Torregrosa, J., Dominguez, J.R., and Peres, J.A. (2001) Oxidation of $p$-hydroxybenzoic acid by UV radiation and by $\mathrm{TiO}_{2} / \mathrm{UV}$ radiation: comparison and modelling of reaction kinetic. J. Hazard Mater., 83, 255-264

Dunn, J., Burgess, D., Leo, F. (1997) Investigation of pulsed light for terminal sterilization of WFI filled blow/fill/seal polyethylene containers. PDA J. Pharm. Sci. Technol., 51, 111-115.

Feldman, H. A. (1977) Comparison of transcriptional linkage of tRNA cistrons in yeast and E. coli by the ultraviolet light technique. Nucleic Acids Res., 4, 2831-2841.

Fine, F., and Gervais, P. (2004) Efficiency of pulsed UV light for microbial decontamination of food powders. J. Food Prot., 67, 787-792.

Harthug, S., and Lingaas, E. (2002) Infection control in hospitals. Tidsskr Nor. Laegeforen, 122, 2317-2318.

Hodgson, M. J., and Flannigan, B. (2001) Occupational respiratory Dosease: Hypersensitivity pneumonitis, Microorganisms in Home and Indoor Work Environments, Flannjgan, B., Samson, R.A and Miller, J.D (eds), CRC press, NY.

Huang, Z., Maness, P-C., Blake, D.M., Wolfrum, E.J., Smolinski, S.L., and Jacoby, W.A. (2000) Bactericidal mode of titanium dioxide photocatalysis. J. Photo. Photo. A: Chemistry 130, 163-170.

Imlay, J.A., Linn, S. (1988) DNA damage and oxygen radical toxicity. Science, 240, 1302-1309.

Ireland, J.C., Klostermann, P., Rice, E.W., and Clark, R.M. (1993) Inactivation of Escherichia coli by titanium dioxide photocatalytic oxidation. Appl. Environ. Microbiol., 59,1668-1670.

Kashige, N., Kakita, Y., Nakashima, Y., Miake, F., and Watanabe, K. (2001) Mechanism of the photocatalytic inactivation of Lactobacillus casei phase PL-1 by titania thin film. Current Microbiol., 42, 184-189.

Ko, G., First, M.W., Burge, H.A. (2000) Influence of relative 
humidity on particle size and UV sensitivity of Serratia marcescens and Mycobacterium bovis BCG aerosols. Tuber Lung Dis., 80, 217-228.

Maness, P.C., Smolinski, S., Blake, D.M., Huang, Z., Wolfrum, E.J., and Jacoby, W.A. (1999) Bactericidal activity of photocatalytic $\mathrm{TiO}_{2}$ reaction: toward an understanding of its killing mechanism. Appl. Environ. Microbiol., 65, 4094-4098.

Miki, A., Kurosu, S., Hayashi, F., Sakakibara, Y., and Shintani, H. (2003a) Determination of microorganisms both airborne and surface from the air at an artificial dialysis in the health care facility. Bokin Bobai 31, 77-84.

Miki A, Kurosu S, Hayashi F, Sakakibara Y, Kato, S, and Shintani H. (2003b) Test of effectiveness of photocatalyst. Clean Technology, 13 (10), 70-73.

Omori, E., Komeda, K., Yokoyama, S., Shintani, H. (2005) On the sterilization of pharmaceutical production area with the mixed gas of peracetic acid and hydrogen peroxide. Bokin Bobai 33 (12) in press.

Sahini-Kardasi, A. (1996) Infection control: do we all have a role to play? EDTNA ERCA J., 22,11-14.

Sakurai, M., Takahashi, R., Fukunaga, S., Shiomi, S., kazuma, K., and Shintani, H. (2003) Several factors affecting ozone gas sterilization. Biocontrol Sci., 8, 69-76.

Setlow, R. B., Carrier, W.L. (1966) Pyrimidine dimers in ultraviolet-irradiated DNA's. J. Mol. Biol., 17, 237-254.

Setlow, R. B., (1966) Cyclobutane-type pyrimidine dimers in polynucleotides. Science, 153, 379-386.

Shibata, H., Ogura, Y., Sawa, Y., and Kono, Y. (1998) Hydroxy radical generation depending on $\mathrm{O}_{2}$ and $\mathrm{H}_{2} \mathrm{O}$ by a photocatalyzed reaction in an aqueous suspension of titanium dioxide. Biosci. Biotech. Biochem., 62, 23062311.

Shintani, H. (1995a) Formation and elution of toxic compounds from sterilized medical products: methylenedianiline formation in polyurethane. J. Biomater. Appl., 10, 23-58.

Shintani, H., Tahata, T., Hatakeyama, K., Takahashi, M (1995b) Comparison of $D_{10}$-value accuracy by the limited Spearman-Karber procedure (LSKP), the StumboMurphy-Cochran procedure (SMCP), and the survivalcurve method (EN). Biomed. Instrum. Technol., 29, 113124.

Shintani, H. (2002) Sterilant, disinfectant, fumigant used in pharmaceutical companies and food companies. Bokin Bobai, 30, 491-500.

Shintani, H. (2003) Present and future of medical device sterilization. Bokin Bobai, 31, 619-623.

Shintani, H., Taniai, E., Miki, A., Kurosu, S., and Hayashi, F. (2004) Comparison of the trapping efficiency of the airborne microorganisms among air samplers. J. Hosp. Infection, 56, 42-48.

Shintani, H., yamase, Y., and yamaguchi, T. (2005) Ethylene oxide gas sterilization method and validation method. Bokin Bobai, 33, 137-149.

Takeshita, K., Shibato, J., Sameshima, T., Fukunaga, S., Isobe, S., Arihara, K., and Itoh, M. (2003) Damage of yeast cells induced by pulsed light irradiatioon. Int. J. Food Microbiol., 85,151-158.

Vincent, J. (2002) Nosocomial infection and outcome. Nutrition, 18, 713-714.

Weist, K., Pollege, K., Schulz, I., Ruden, H., and Gastmeier, P. (2002) How many nosocomial infections are associated with cross-transmission? A prospective cohort study in a surgical intensive care unit. Infect Control Hosp. Epidemiol., 23, 127-132.

Wolfrum, E.J., Huang, J., Blake, D.M., Maness, P.C., Huang, Z., Fiest, J., and Jacoby, W. A. (2002) Photocatalytic oxidation of bacteria, bacterial and fungal spores, and model biofilm components to carbon dioxide on titanium dioxide-coated surfaces. Environ. Sci. Technol., 36, 3412 3419.

Yasui, A., and McCready, S. J. (1998) Alternative repair pathways for UV-induced DNA damage. Bioassays, 20, 291-297. 